\title{
SECOND PANEL DISCUSSION
}

[This panel and discussion was intended to concentrate on observational connections and future observational directions. Panel members were P. Conti, R.M. Humphreys, A. Maeder, O. Stahl, and N.R. Walborn, with A.F.J. Moffat acting as moderator or chairman. Remarks by panel members and other participants are given here in condensed form, partially reworded for brevity and clarity.]

Conti: Concern has been expressed throughout this meeting as to the definition of an LBV. While these letters originally meant Luminous Blue Variables, we have seen during these four days that some are not blue, some are not luminous, and some are not (yet) variable. As far as proposing a 'legal' definition, I am somewhat mindful of the judge who when asked to define pornography responded that he couldn't but "knew it when he saw it." In that spirit, I would like to suggest that LBV stars be considered Little Bit Violent stars. We would all agree that those objects showing this kind of behavior are LBV's. We probably also need to consider LBV candidates.

What is the role of LBV's in massive star evolution? They are similar to WN stars in having enhanced $\mathrm{He}$ and $\mathrm{N}$ abundances, comparable luminosities, masses, and massloss rates, and irregular variability, along with ejections of ring nebulae in several instances. While all LBV's have hydrogen, only some of the WN's have evidence of this element being present. It seems safe to conclude that some LBV's become WN stars although it is by no means clear that all do, nor that all W-R stars have gone through an LBV phase. It is premature to associate any W-R subtype with a predecessor LBV phase, even though a connection to WN8 stars is tempting. I would like to emphasize that there is no relation (yet) between any evolution model and any $W-R$ subtype, especially since the latter have very heterogeneous properties.

I would like now to pose some questions: Are there different scenarios for 'bright' (R 127) and 'faint' (R 71) LBV's, above and below the red supergiant limit? Will all stars with anomalous emission lines eventually become LBV's? Does the core physics or the surface composition terminate the LBV phase? What role do binaries play in the LBV phases?

Humphreys: Not all luminous emission-line stars are LBV's. We have seen in this colloquium that there are actually very few known LBV's. Numbers presented here suggest that the duration of this stage is very short, only of the order of 20000 years. This is a short but physically important state in the evolution of very massive stars.

A variety of objects are near the luminosity/stability limit in the upper H-R diagram - LBV's, B[e] supergiants, unstable cool hypergiants, and 'normal' mass-losing $O, B$, and A-type supergiants. What are the relationships among these different objects, and what is the relation between the appearance of the upper H-R diagram and the cause of the instabilities?

K. Davidson et al. (eds.), Physics of Luminous Blue Variables, 249-254.

(1) 1989 by Kluwer Academic Publishers. 
The temperature-dependent luminosity limit for hot stars and the upper luminosity boundary for the cool hypergiants are related. The observed limit for the hot stars is most likely due to radiation pressure and the opacity-dependent Eddington limit. The Kudritzki et al. 'hydrogen recombinator' (Kudritzki's word!) in the stellar atmosphere is a possible trigger. Where the opacity reaches a maximum at $8000-10000 \mathrm{~K}$, the Eddington luminosity may be at its minimum. Below this luminosity, stars can evolve to become red supergiants or hypergiants. We also know from de Jager's studies of the atmospheres of cool hypergiants that these stars' surfaces can become unstable because of turbulent pressure. Thus the turbulent pressure gradient in cool atmospheres, as well as the radiation pressure obstacle to redward evolution of hotter stars, sets an upper limit to luminosities in the cool part of the H-R diagram. Based on observations of nearby galaxies, this limit is near a bolometric absolute magnitude of about $\mathbf{- 9 . 5}$. Three very luminous A-type supergiants with bolometric magnitudes around -10 thus occupy a critical place in the H-R diagram: Cyg OB2 \#12, HD 33579, and B 324 in M33. It is uncertain whether they can evolve to the red supergiant stage. Maeder's density inversion may play a role in stars like these.

The $B[e]$ supergiants appear to be caused by a different type of instability. The observations show that they have relatively dense equatorial disks. This must be due to high mass loss at their equators, possibly caused by rotation or binary interaction. I doubt if there is any direct evolutionary connection with the LBV's.

One of our most important observational tasks is to identify and study more LBV's to improve our database on these very rare stars.

Stahl: Since we now apparently all know what an LBV is, we may turn to other stars.

In the upper H-R diagram we find not only LBV's, but also 'normal' stars and many other emission-line stars which have not (yet?) shown any outbursts and thus cannot be classified as LBV's. What is the relation of these stars to the LBV's? It is likely that that many of them are LBV's in quiescent phases. Af ter all, R127 was detected behaving as an LBV only about five years ago; before that outburst it was a 'normal' Ofpe/WN9 star. This makes the Ofpe/WN9 stars especially promising LBV candidates, although some of them appear to be much fainter than R 127 .

Nevertheless, it may be that some of these stars never show outbursts. The $B[e]$ supergiants, for example, have been suggested to be a completely different class of emission-line stars, which do not show any outbursts. These stars may have disks formed by rapid rotation. However, observationally, it has yet to be shown that the $B[e]$ supergiants do indeed rotate fast.

The role of binarity also still has to be clarified. So far only one binary, R 81 , is known among the luminous emission-line stars. This star has not shown any outburst so far and it even has unusually small micro-variations. So the roles of rotation and binarity are unclear for LBV's and need to be attacked observationally.

Walborn: An interesting morphological challenge would be to devise criteria discriminating large red tomatoes from large red apples, while suggesting an evolutionary relationship between small green apples and large red ones, under the condition that one is not allowed to approach close enough to bite! The opposite might be more readily inferred. Moreover, small green tomatoes and large green apples also exist, not to mention crab apples! The problems of classifying and interpreting the amazing array of envelope phenomena that hot massive stars can produce are rather analogous. We 
are far from understanding the physical reasons for all this diversity, and the underlying generic relationships. I would like to highlight some aspects of three specific issues.

1. Eta Carinae: Usually we think of the LBV phase as intermediate between $\mathrm{O}$ and WR states, which may apply to the typical LBV. However, I suggest that $\eta \mathrm{Car}$ is a post-WN-A (narrow-line WN) object. This star is unique among known LBV's in terms of its location within a giant HII region associated with $\mathrm{O} 3$ and WN-A stars of initial masses at least $100 M_{\odot}$, and in terms of the magnitude of its outburst amplitude (due to a higher effective temperature and bolometric correction?). The spectroscopic progression from $\mathrm{O} 3 \mathrm{~V}$ through $\mathrm{O} 3$ If to $\mathrm{WN}-\mathrm{A}$ is very smooth; it is difficult to imagine the chaotic $\eta$ Car phase interpolated between $\mathrm{O} 3$ If and $\mathrm{WN}-\mathrm{A}$, when the relative appearance of these two kinds of spectra strongly suggests simply an increase in wind density between them. The WN-A stars have the highest hydrogen content and luminosities of all WR's, and it is plausible that they are produced by steady mass loss from the most massive $O$-type stars. It is noteworthy that no $\eta$ Car counterpart is observed in 30 Doradus, despite the much larger early-O and WR populations there. Possibly this indicates a very short time-scale for the $\eta$ Car phenomenon. If the suggestive similarities between the $\eta$ Car nitrogen-rich ejected knots and the quasi-stationary flocculi in Cas A are significant, then $\eta$ Car will become a supernova on a time-scale shorter than $10^{4}$ years. The only caveat to the foregoing is the question whether the $\eta$ Car phase is naturally experienced by all of the most massive stars, or corresponds to some pathological event such as a close binary interaction. If the former is the case, the suggestion is that the WR and LBV phases bear a fundamentally different relationship at the highest stellar masses, compared to their roles at somewhat lower masses.

2. Concerning the Ofpe/WN9 -- LBV connection: Are all Ofpe/WN9 objects quiescent LBV's? Conversely, are all LBV's (either in some mass-luminosity range or simply all) Ofpe/WN9 stars when they are at minimum? R71 definitely has a lower luminosity than other LBV's in the LMC, but some of the Ofpe/WN9's also have relatively low luminosities e.g., BE 381). In this case P Cyg and S Dor would be in extended intermediate or maximum states, and their spectra at minimum have not yet been observed. The P Cyg light curve shown at this colloquium by de Groot indicates both brighter and fainter states during the 17 th Century, with an intermediate light level since, consistent with the 'intermediate' B-type spectrum currently observed. Of course, the foregoing questions bear directly on the reality of the proposed LBV 'instability strip;; if all are actually Ofpe/WN9 at minimum, then the high-temperature limit of $\mathrm{LBV}$ excursions in the H-R diagram would be vertical as is the low-temperature one.

Some further tomato-vs.-apple questions: Does the behavior of HDE 269582 imply that some other Bpe objects in the LMC are really Ofpe/WN9's? If so, they are becoming remarkably numerous. Are some WN8, Ofpe/WN9, and LBV objects near or below the luminosity limit for red supergiants in the H-R diagram actually post-redsupergiants? Possible examples are P Cyg (see Lamers et al., Astron. Astrophys. 123, AG Car and R71 (McGregor et al., Astrophys. J. 329, 874), HD 96548 (Smith et al., M.N.R.A.S. in press), and HDE 269227 (Ofpe/WN9--RSG spectrum composite, Schmutz et al. in these proceedings); in several cases the post-red-supergiant interpretation is suggested by the characteristic of the circumstellar nebulae. Finally, we need more observational evidence for the putative $\mathrm{O} \rightarrow \mathrm{LBV} \rightarrow$ WNE/WC evolutionary sequence, such as physical associations among objects in these relatively short phases.

3. Disarray on the Late WN Sequence: For some time we have been used to thinking in terms of the 'WR phenomenon' in the context of massive objects and planetary 
nebula nuclei with superficially similar spectra. However, it may be necessary to emphasize this concept within the Population I sample itself: there may be qualitatively different physical origins for objects in adjacent subclasses and even within a given subclass. For instance, most WN6/7-A objects are in giant $\mathrm{HII}$ regions or young associations and have very high luminosities $\left(\mathrm{M}_{\mathrm{V}} \sim-6.5\right.$ to -7.5$)$; CQ Cep is not, but is a close binary, so it provides a poor paradigm for this subclass. Most WN8 objects are not in giant HII regions and have lower luminosities; some may be post-red-supergiants. The nature of the Ofpe/WN9 class in the LMC and its relation to the LBV's has been amply discussed here. Some galactic objects have been proposed as counterparts to the LMC Ofpe/WN9 class, and other isolated objects have been classified as WN10 or WN11 in the literature, but without a systematic morphological analysis relative to the existing WN sequence. A larger sample of such objects with homogeneous data and a systematic analysis are required in order to satisfactorily define the late WN sequence in the future. Several such objects have been presented at this meeting, a welcome development.

Maeder [points excerpted from a tape recording of his talk]: Can we understand LBV's without understanding evolution? I think the answer is no. Can we understand evolution without understanding LBV's? I think the answer is no. ... Here I wish to emphasize the relations between model ingredients, model outputs, and observations.

A model uses only a few equations but many ingredients: nuclear reactions and weak interaction parameters, opacities, boundary conditions, initial abundances, convection and overshooting, mass loss, diffusion, rotation, binarity ... When the model outputs agree with observations, everyone is happy but we do not make much further progress. When they disagree, people are not so happy, but future progress results. To make such progress, we must identify which particular features of the model are responsible for the disagreement; maybe the disagreement tells us that some assumed ingredient is wrong. But there is predictive power in models, too; for instance, LBV's may be gamma-ray sources because of ${ }^{26} \mathrm{Al}$ in their atmospheres and ejecta ...

Physical quantities represented in models and observed quantities are of ten different entities. To improve the connections, it would be good to obtain very detailed observations (photometry, abundances, pulse periods, etc.) of stars within clusters. Not all abundances constrain models equally well; e.g., for WN stars the $\mathrm{C} / \mathrm{N}$ ratio is more or less the same whatever the evolution, but $\mathrm{C} / \mathrm{H}$ is a very constraining quantity. Finally, if everything could be predicted accurately from theory, then we would not have the pleasure of attending meetings on the topic!

\section{General discussion:}

Walborn: Roberta mentioned Cyg OB2 \#12. I think that the association membership of this star is questionable; its late-B spectral type is incompatible with the of character of the association. It might not be as distant or as luminous as generally assumed. We have to be especially careful about distances in Cygnus and Carina, where we're looking along spiral features.

Humphreys: \# 12 is the most highly reddened star in that association -Walborn: But there is a strong gradient in the reddening in that direction. 
Wolf: Many years ago I analyzed HD 33579, another of the luminous stars that Roberta mentioned. The methods would certainly not be considered state-of-the-art now, but the $\log g$ that I found indicated a low mass, of the order of $25 M_{\odot}$. This indicates that HD 33579 is very evolved, it has lost much mass.

Cassinelli: With regard to rotation - (1) Is the observational situation regarding $v$ sin $i$ going to improve for the $\mathrm{B}[\mathrm{e}]$ stars? $\mathrm{R} 126$ is especially frustrating because there's evidence for rapid rotation but no value of $v \sin i$. (2) Peter Conti mentioned that both LBV's and WR stars have ring nebulae. Did you mean to imply that they are spherically symmetric?

Walborn: Lacking good photospheric absorption lines, it is hard to see how we can observe $v \sin i$. Realizing this, in my own mind there is some doubt about the rapid rotations.

Conti: I was not making any assertion about spherical nebulae. In most cases with hot central stars they are not symmetric. At least part of the reason could be rotation of the central object, and another cause may be the non-homogeneous interstellar environment into which expansion occurs.

Niemela: NGC 6888 has been mentioned in this connection. However, it is mostly swept-up material, maybe not directly comparable to the AG Car nebula.

Conti: Perhaps those two nebulae are not directly comparable. But what happens to an AG-Car-like object after the central star becomes a WN with a strong wind? Then, too, more material might be swept up.

Niemela: Just what is an anomalous O-emission-line star?

Conti: Two kinds of 'slash' stars were introduced by Walborn in the 1970's. Ofpe/ WN stars have anomalous SiIV features and also other emission features not normally seen in Of-type stars. The other kind has features intermediate between types $\mathrm{O}$ and WN. R 127 was an example of the first class; maybe the other class can also lead to LBV's.

Walborn: R 127 was not only an example of the Ofpe/WN9 class, it was a prototype of that class. Still, the hypothesis that Ofpe/WN9's all become LBV's may be an extreme one.

Shore: But what other trick do we have for finding LBV's?

Walborn: We have to wait for their outbursts.

Shore: That'll take a lot of GSL's ('graduate-student-lifetimes').

Humphreys: The Hubble-Sandage variables offer hope that we can expand the sample of galaxies, especially if the Space Telescope becomes available. For the known $\mathrm{H}-\mathrm{S}$ variables, older photometric data is available to supplement newer spectroscopic data.

Shore: Although we haven't had enough time for this point here, there is something very strange about the space distribution of the massive stars. They tend to appear in compact groups, groups much smaller than associations. Also, maybe we tend to look for LBV's where they are easy to find, say in fields that are not too crowded. are LBV's distributed uniformly in galactic disks? Abundance gradients may be relevant here.

Humphreys: In M33 the known LBV's are all on one side, and not very far out. Var $A$ is farther out, in the outermost arm. 
Moffat: The Upper Limit in the H-R diagram is fuzzy. How fuzzy?

Humphreys: Here we are definitely in the realm of small-number statistics, we don't have any way to know exactly where to draw the line, and no solution has been proposed during this colloquium. We can define it as the actual upper envelope, connecting just several of the most luminous stars. Or we can draw it about half a magnitude lower where there are more stars. A better definition requires both better statistics and a more definite theoretical background.

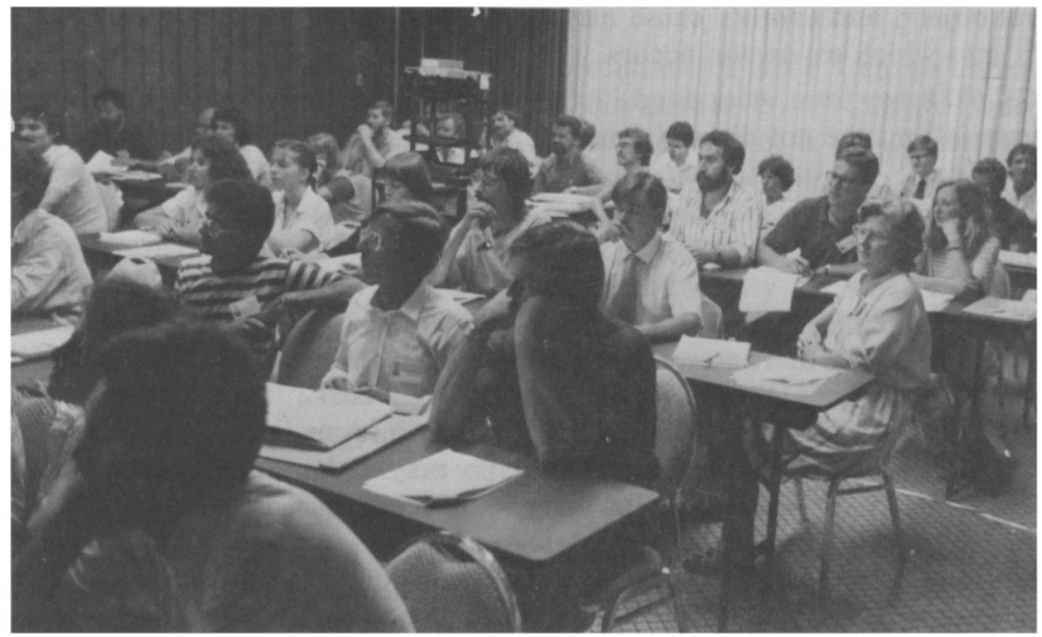

